\title{
A TWIST in the fate of human osteoblasts identifies signaling molecules involved in skull development
}

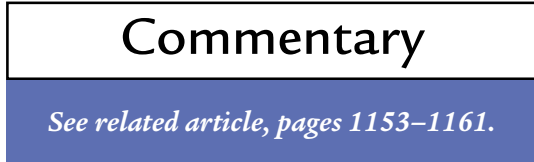

\author{
Ethylin Wang Jabs
}

Center for Craniofacial Development and Disorders, Institute of Genetic Medicine, Departments of Pediatrics, Medicine, and Plastic Surgery, The Johns Hopkins University School of Medicine, Children's Medical and Surgical Center 1004, 600 North Wolfe Street, Baltimore, Maryland 21287-3914, USA. Phone: (410) 955-4160; Fax: (410) 955-0484; E-mail: ewjabs@jhmi.edu.

The twist gene was first recognized by the Nobel Prize-winning laboratory of Nüsslein-Volhard (1) and described in Drosophila as a zygotic gene required for dorsoventral patterning (2). Along the dorsoventral axis during the blastoderm stages of Drosophila, the most ventral nuclei constitute the mesodermal anlage which gives rise to cells that invaginate at gastrulation. With the loss of twist expression, homozygous mutant embryos fail to develop a mesoderm layer, resulting in few if any mesodermally derived internal organs. The head segments appear everted due to abnormal head involution, and the anterior end of the embryo is "twisted" in the egg.

In mice, Twist expression also is initially observed along the dorsoventral gradient and then in the mesoderm and neural crest cell derivatives (3). Later, Twist is found in the somites, head mesenchyme, aortic arches, branchial arches, limb buds, and mesenchyme underneath the epidermis (4). Twist is expressed in the undifferentiated cells committed to muscle and cartilage development and inhibits their differentiation. Its transcripts are present in primary osteoblastic cells of the newborn mouse calvaria (5). Mouse embryos homozygous for the disruption of the Twist gene exhibit defects in the head mesenchyme, failure of neural tube closure in the cranial region, and pyknotic nuclei found in cranial nerves (6). Heterozygous mice display skull defects of poorly developed squamosal bones and of overdeveloped interparietal bones and limb abnormalities (7).

The conservation of the Twist mutant phenotype also extends to humans. The heterozygous loss of the TWIST gene or its function results in a common autosomal dominant syndrome, Saethre-Chotzen, which is characterized by craniosynostosis, facial dysmorphisms, and hand and foot abnormalities $(8,9)$. Of these developmental abnormalities, craniosynostosis - the premature fusion of cranial sutures - is the most notable. Among the cranial sutures, the coronal suture across the width of the skull is most often prematurely synostosed, leading to a brachycephalic skull one that is box-shaped and of shortened length. Mineralization of the cranium normally occurs directly from membrane-derived paraxial mesoderm. Ossification centers coalesce to form bones, and their osteogenic fronts meet to induce sutures along their lines of approximation. The skull continues to grow by appositional growth at the suture, with deposition of premineralized bone matrix along the suture margins. Several hypotheses have been proposed that premature synostosis is due to the abnormal development of the mesodermal blastema, accelerated bone growth, and/or increased differentiation. The discovery of the new disease gene TWIST generates an important tool that potentially could help in elucidating the signaling pathway and pathogenesis involved in craniosynostosis. A study reported in this issue of the JCI is evidence that this potential is being realized. Yousfi and colleagues from the Marie laboratory (10) identified TWIST target genes using human mutant calvaria osteoblastic cells from a child with Saethre-Chotzen syndrome. This individual carries a previously reported and apparently recurrent point mutation in TWIST that introduces a premature stop codon $(8,9,11)$. The product of this Y103X mutant allele would be predicted to be truncated upstream of the basic helix-loop-helix (bHLH) domain, which is normally required for dimerization and for DNA binding. However, since the patient's osteoblasts showed decreased TWIST mRNA and protein lev-

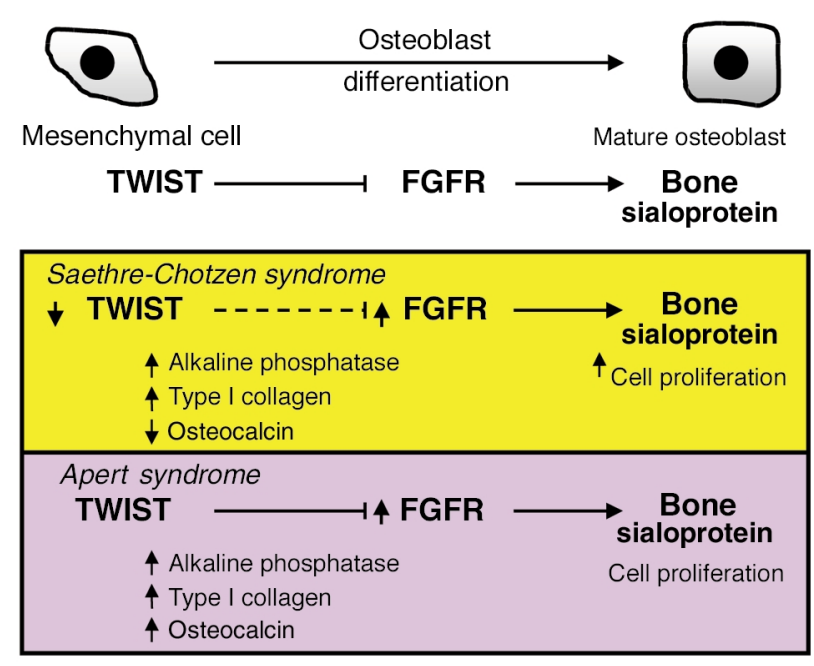

\section{Figure 1}

Elements involved in the molecular pathway of sutural osteoblast differentiation (modified from ref. 15). Top: normal cellular differentiation and relationship of TWIST and FGFR signaling. Bottom: altered gene expression in TWIST Y103X mutant osteoblasts from a SaethreChotzen syndrome patient and in FGFR2 S252W mutant osteoblasts from an Apert syndrome patient. Bone sialoprotein is a mature osteoblast marker. 
els, it seems likely that mRNAs carrying this mutation do not survive nonsensemediated decay and that the Y103X allele is a true null. In vitro and in vivo experiments with TWIST mutant cells showed increased cell growth and ability to form bonelike nodular structures. These cells and aggregates exhibited increased expression of osteoblastic markers, alkaline phosphatase, and type I collagen, independent of cell growth. However, TWIST mutant cells showed reduction of osteocalcin mRNA expression during osteogenesis. The altered expression of these osteoblastic genes with craniosynostosis is consistent with previously published observations $(5,12$, 13). Overexpression of TWIST by transfection studies in osteosarcoma cells may inhibit osteoblast differentiation (12), and reduction of murine Twist expression occurs with osteoblastic maturation (5). Osteocalcin-deficient mice have increased bone formation (13). Therefore, the significance of the findings from the Marie laboratory (10) is that the premature ossification of cranial sutures in Saethre-Chotzen syndrome patients with TWIST mutations is caused by increased osteoblastic cell proliferation and differentiation (Figure 1).

\section{Finding other candidate genes for craniosynostosis syndromes}

With these findings, the signaling pathways that lead to craniosynostosis in Saethre-Chotzen and other syndromes is only beginning to come to light. Several other genes recognized as targets of TWIST or involved in osteogenesis offer additional candidates that should be tested for their roles in osteoblast development. Most relevant are the DERMO1 and ID genes, which code for other bHLH proteins and TWIST dimerization partners. Both are expressed in osteoblasts, and, like TWIST, DERMO1 and ID may be negative regulators of differentiation of osteoblasts $(5,14-16)$, although ID is known to inhibit bHLH factors. Msh2 and DFR1 have also been proposed as targets because mesodermal expression of these genes is disrupted in the twist mutant embryos of Drosophila $(17,18)$.

Further evidence that these human homologues may be involved in the signaling pathway for skull development has come from genetic studies. For example, the human homologue of msh2, MSX2, is typically expressed in neural crest-derived mesenchyme of the branchial arches and the developing calvarial sutures (19). When MSX2 is mutated, it affects skull development such that the phenotypic outcome is finely calibrated by its expression level. Overexpression of MSX2 due to a missense mutation in its homeodomain results in Boston-type craniosynostosis (19), whereas loss of function mutations result in parietal foramina, "holes in the skull" (20). The presence of binding sites for MSX, as well as HLH proteins at the Osteocalcin promoter, indicates potential interactions $(21,22)$. The mammalian homologues of DFR1, the FGF receptor genes (FGFRs), are also implicated in craniosynostosis. FGFRs may mediate a signal downstream of Twist because murine Twist expression precedes that of Fgfr genes during the initiation of the coronal suture (23). Fgfr2 and Twist expression patterns overlap at the most immature proliferating osteogenic tissues. Fgfr2 is a marker of proliferating uncommitted cells, not of osteogenic differentiation. An activating mutation has been detected in FGFR3 for some cases that phenotypically resemble Saethre-Chotzen syndrome (11) and in FGFR1 and FGFR2 for other craniosynostosis syndromes that are clinically distinct from Saethre-Chotzen syndrome.

Apert syndrome, one of the most phenotypically severe craniosynostosis conditions, results from FGFR2 mutations that cause altered ligand specificity. Preosteoblast calvaria cells from an Apert syndrome fetus have increased alkaline phosphatase and type I collagen expression with mineralized matrix formation but normal cell growth, suggesting that an increased maturation rate of cells in the osteoblastic lineage leads to premature ossification (Figure 1 ; see also ref. 24). The increased differentiation rate of osteoblasts in both Apert and Saethre-Chotzen syndromes results in coronal synostosis. The relatively slower rate of osteoblast cell growth in the Apert syndrome patient, as compared with the Saethre-Chotzen syndrome patient, may account for the presence of a wide open gap of the skull bones in Apert but not in SaethreChotzen syndrome. This gap, which extends from the forehead back to the occiput, is created by the failure of fusion along the medial portion of these sutures. Another craniosynostosis condition, Pfeiffer's syndrome, associated with a missense mutation in FGFR1, results in increased expression of CBFA1, with premature fusion of calvarial sutures (25). CBFA1 encodes an osteoblast-specific transcription factor that binds to the Osteocalcin promoter and regulates osteoblast differentiation. Other osteoblastic genes of interest that have not been implicated in craniosynostosis include the bone morphogenetic proteins, a family of TGF- $\beta$ homologues. BMP2 upregulates Id (16) and can induce ectopic bone formation in vivo, osteoblastic differentiation, and expression of osteoblastic genes, such as alkaline phosphatase (26). TGF- $\beta$ s 1-3 are also expressed in the mouse sutures (27) and may be involved in this developmental pathway.

With identification of more human genes involved in sutural development or craniosynostosis and with access to mutant osteoblasts, more information about DNA and protein interactions will emerge from genetic and biochemical experimentation. Additional extracellular components, such as hormones, growth factors, and cytokines that modulate skull development, will also undoubtedly be discovered, yielding a wealth of "players" to study the individual signaling pathways involved in each of the more than 100 known craniosynostosis conditions.

\section{Acknowledgments}

This work was supported by NIH grants P60 DE13078 and R01 DE11441.

1. Nüsslein-Volhard, C., Wieschaus, E., and Kluding, H. 1984. Mutations affecting the pattern of the larval cuticle in Drosophila melanogaster I. Zygotic loci on the second chromosome. Wilhelm Roux Arch. Dev. Biol. 193:267-282.

2.Simpson, P. 1983. Maternal-zygotic gene interactions during formation of the dorsoventral pattern in Drosophila embryos. Genetics. 105:615-632.

3. Stoetzel, C., Weber, B., Bourgeois, P., BolcatoBellemin, A.L., and Perin-Schmitt, F. 1995. Dorso-ventral and rostro-caudal sequential expression of M-twist in the postimplantation murine embryo. Mech. Dev. 51:251-263.

4. Fuchtbauer, E.M. 1995. Expression of M-Twist during postimplantation development of the mouse. Dev. Dyn. 204:316-322.

5. Murray, S.S., et al. 1992. Expression of helixloop-helix regulatory genes during differentiation of mouse osteoblastic cells. J. Bone Miner. Res. 7:1131-1138.

6. Chen, Z.F., and Behringer, R.R. 1995. twist is required in head mesenchyme for cranial neural tube morphogenesis. Genes Dev. 9:686-699.

7. Bourgeois, P., et al. 1998. The variable expressivity and incomplete penetrance of the twist-null heterozygous mouse phenotype resemble those of human Saethre-Chotzen syndrome. Hum. Mol. Genet. 7:945-957.

8. Howard, T.D., et al. 1997. Mutations in TWIST, a basic helix-loop-helix transcription factor, in Saethre-Chotzen syndrome. Nat. Genet. 15:36-41.

9. El Ghouzzi, V., et al. 1997. Mutations of the 
TWIST gene in the Saethre-Chotzen syndrome. Nat. Genet. 15:42-46.

10. Yousfi, M., Lasmoles, F., Lomri, A., Delannoy, P., and Marie, P.J. 2001. Increased bone formation and decreased osteocalcin expression induced by reduced Twist dosage in Saethre-Chotzen syndrome. J. Clin. Invest. 107:1153-1161.

11. Paznekas, W.A., et al. 1998. Genetic heterogeneity of Saethre-Chotzen syndrome, due to TWIST and FGFR mutations. Am. J. Hum. Genet. 62:1370-1380.

12. Lee, M.S., Lowe, G.N., Strong, D.D., Wergedal, J.E., and Glackin, C.A. 1999. TWIST, a basic helix-loop-helix transcription factor, can regulate the human osteogenic lineage. J. Cell. Biochem. 75:566-577.

13. Ducy, P., et al. 1996. Increased bone formation in osteocalcin-deficient mice. Nature. 382:448-452.

14. Tamura, M., and Noda, M. 1999. Identification of DERMO-1 as a member of helix-loop-helix type transcription factors expressed in osteoblastic cells. J. Cell. Biochem. 72:167-176.

15. Lee, M.S., Lowe, G., Flanagan, S., Kuchler, K., and Glackin, C.A. 2000. Human Dermo-1 has attributes similar to twist in early bone development. Bone. 27:591-602.
16. Rice, D.P., et al. 2000. Integration of FGF and TWIST in calvarial bone and suture development. Development. 127:1845-1855.

17. Bodmer, R., Jan, L.Y., and Jan, Y.N. 1990. A new homeobox-containing gene. Msh-2 is transiently expressed early during mesoderm formation of Drosophila. Development. 110:661-669.

18. Shishido, E., Higashijima, S., Emori, Y., and Saigo, K. 1993. Two FGF-receptor homologues of Drosophila: one is expressed in mesoderma primordium in early embryos. Development 117:751-761.

19. Jabs, E.W., et al. 1993. A mutation in the homeodomain of the human MSX2 gene in a family affected with autosomal dominant craniosynostosis. Cell. 75:443-450

20. Wilkie, A.O., et al. 2000. Functional haploinsufficiency of the human homeobox gene MSX2 causes defects in skull ossification. Nat. Genet. 24:387-390.

21. Heinrichs, A.A., et al. 1993. Identification and characterization of two proximal elements in the rat osteocalcin gene promoter that may confer species-specific regulation. J. Cell. Biochem. 53:240-250.

22. Tamura, M., and Noda, M. 1994. Identification of a DNA sequence involved in osteoblast-spe cific gene expression via interaction with helix loop-helix (HLH)-type transcription factors. $J$ Cell Biol. 126:773-782.

23. Johnson, D., Iseki, S., Wilkie, A.O., and MorrissKay, G.M. 2000. Expression patterns of Twist and Fgfr 1, -2, and -3 in the developing mouse coronal suture suggest a key role for Twist in suture initiation and biogenesis. Mech. Dev. 91:341-345

24. Lomri, A., et al. 1998. Increased calvaria cell differentiation and bone matrix formation induced by fibroblast growth factor receptor 2 mutations in Apert syndrome. J. Clin. Invest. 101:1310-1317.

25. Zhou., Y.X., et al. 2000. A Pro250Arg substitution in mouse Fgfr 1 causes increased expression of Cbfa1 and premature fusion of calvarial sutures. Hum. Mol. Genet. 9:2001-2008.

26. Wozney, J.M., et al. 1988. Novel regulators of bone formation: molecular clones and activities. Science. 242:1528-1534.

27. Opperman, L.A., Nolen, A.A., and Ogle, R.C. 1997. TGF- $\beta 1$, TGF- $\beta 2$, and TGF- $\beta 3$ exhibit distinct patterns of expression during cranial suture formation and obliteration in vivo and in vitro. J. Bone Miner. Res. 12:301-310. 